\title{
Early Experience of Oncological Reconstructive Surgery at Dharmais National Cancer Center
}

\author{
Rian Fabian Sofyan*, Bayu Brahma, Iskandar, Putri Naura \\ Department of Surgical Oncology, Dharmais Cancer Hospital - National Cancer Center, Jakarta, Indonesia
}

\section{ARTICLE INFO}

Received : 25 January 2021

Reviewed : 12 March 2021

Accepted : 21 May 2021

Keywords:

advanced-stage cancer, free flap, microsurgery, oncology reconstructive, survival

*Corresponding author:

Rian Fabian Sofyan

Department of Surgical Oncology, Dharmais Cancer Hospital - National Cancer Center, Jakarta, Indonesia rianfabian@gmail.com

\section{A BSTRACT}

Background: Oncological reconstructive surgery is a new paradigm in which it combines oncologic principles with reconstruction techniques. This study aims to present patients' characteristics who had undergone oncological reconstructive surgery at the Department of Surgical Oncology, Dharmais National Cancer Center.

Methods: This descriptive study identified patients' characteristics who underwent oncological reconstructive surgery, their cancer types, and their therapies. All data were obtained from surgery registration and medical records from January 2019 to January 2020. Data were then presented in number and percentage.

Results: A total of 174 patients had undergone oncological reconstructive surgery within one year. The mean age of all patients was $48.2 \pm 16.7$ years old. Most patients were female $(72.1 \%)$, with breast cancer making up most cases (43.7\%). Most of the participants also underwent mastectomy $(42.1 \%)$, which was later followed by various reconstruction types. Sixty patients received free tissue transfer with microsurgery (34.4\%), of which head and neck cancer constituted most cases. In addition, the most common donor site for the free flap was the Anterolateral Thigh (41.7\%). Of all 174 patients, $75.2 \%$ presented with locally advanced cancer, and $17.8 \%$ had metastatic cancer. Within one year of follow-up, the survival rate was $87.4 \%$, while local recurrence was $3 \%$.

Conclusions: The oncological reconstructive surgery approach plays a therapeutic and reconstructive role, and such might be the preferred method of choice for patients presenting with advanced-stage cancer.

\section{INTRODUCTION}

Oncological reconstructive surgery is a new paradigm in which it combines oncologic principles with reconstruction techniques. The goals of oncological reconstructive surgery include complete removal of the lesion, clear margins, good to excellent reconstruction results, and one-time operating to perform the definitive procedure [1-3].

In our institution, most patients presented with locally advanced cancer, in which the tumor was large relative to the organ size, or sometimes, it was located in an unfavorable position. The excision performed in this area might lead to significant deformity and functional impairment. Thus, the reconstruction of extensive defects after resection has always been challenging [1,2].

In this case, oncological reconstructive surgery combines surgical oncological conventions in a reconstructive manner. It is defined as the immediate reshaping of the remaining tissue, using different forms of remodeling after wide excision of the primary lesion. It can range from simple flap advancement, local flap to free flap taken from various body areas. The combination of these two techniques is intended to avoid poor wound appearance and functional impairment of the organ itself after wide excision. This type of surgery pushes the operative limits of cancer therapy while maintaining the best possible oncological and wound outcomes [4,5]. A retrospective study of 484 patients with T3-T4 oral squamous cell carcinoma conducted at MD Anderson Cancer Center revealed that oncological reconstructive surgery, namely reconstructive microsurgery, contributed to better oncologic outcomes. Other findings included reduced morbidity and improved function following oral cancer treatment [5]. 
Therefore, in this paper, we would like to emphasize the advantages of oncological reconstructive surgery in cancer therapy by outlining our surgery results. All patients were treated at the Dharmais National Cancer Center.

\section{METHODS}

From January 2019 until January 2020, a total of 174 patients underwent oncological reconstructive surgery using different surgical techniques. We retrospectively analyzed the medical records of 174 patients diagnosed with various cancers. The patients' medical records were analyzed, and the following variables were recorded: gender, age, comorbidities, primary tumor site, cancer stage, flap type, and surgery types. Besides, the ethical clearance was obtained from the ethics commission of health research in Dharmais National Cancer Center with the Number 160/KEPK/XII/2020.

All surgical procedures were performed with a twoteam approach. One team was responsible for tumor resections, while the other was responsible for the defect reconstructions, including flap harvesting, preparing the vascular recipient, and microvascular anastomosis.

All patients were treated by surgery to remove the tumor en bloc and followed with immediate reconstruction. Surgical resection at the primary site was aimed to achieve at least a $5 \mathrm{~mm}$ microscopic margin of normal tissue clearance beyond the tumor on the frozen section. Intraoperative assessment of the surgical margin at the primary site was performed employing frozen section analysis, which guided the requirement for further resection.

The patients were then followed up clinically every day right after the surgery and eventually once a month after discharge during the first six months. All participants were followed for at least twelve months or until death. During the twelve-month follow-up, we assessed the survival of these patients and the local recurrence of cancer. Survival duration was defined as the interval from the surgical cancer treatment to the day of the death from the disease or one year after. Meanwhile, local recurrence of the cancer was defined as a reappearance of cancer in the primary site or regional site, confirmed by the clinical and radiological examination.

Descriptive statistics were reported as a count or as a proportion. The results were also presented as mean \pm standard deviation (SD) values.

\section{RESULTS}

\section{Patient characteristics and demographics}

Table 1 demonstrates the overall patient characteristics. One hundred and seventy-four patients underwent oncological reconstructive surgery. The mean age of the study population was $48.2 \pm 16.7$ years. Besides, seventy-two percent of the patients were female $(n=125)$. Of all comorbidities recorded preoperatively, the one with the highest prevalence was anemia, which occurred in $66.7 \%$ of patients. The most common type of cancer in this study was breast cancer, which made up $43.7 \%$ of the cases $(n=76)$.

Head and neck cancer occurred in forty-four patients (25.3\%). Twenty-seven patients (15.5\%) had skin cancer, of which five were melanoma, while the rest was nonmelanoma skin cancer, consisting of basal cell carcinoma and squamous cell carcinoma. Soft tissue tumors made up twenty-five patients $(14.4 \%)$, with sarcoma as the most prevalent type. Meanwhile, thyroid cancer accounted for two patients (1.7\%).

Among all patients, only $7 \%(n=12)$ were detected at an early stage of cancer, while the rest presented locally advanced (75.2\%) and metastases (17.8\%). The clinical staging was carried out in accordance with the eighth edition of the UICC/AJCC TNM staging system.

\section{Surgical procedure}

The main types of surgery performed to remove the tumor vary and depend on the organ of tumor origin. However, most of our surgeries were aggressive. Mastectomy was performed in seventy-three patients (42.1\%), and wide excision was performed in fifty-four patients (31.1\%) (Table 2). Meanwhile, in head and neck cancer cases, we often had to cut the tumor structure. The extent of cutting also varied with tumor status and operator decisions.

\section{Reconstruction types}

The following are the flap types based on the tissue transfer mode. The local flap was performed in ninety-seven patients (55.6\%), while the free flap was performed in sixty patients $(34.4 \%)$ (Table 1$)$. We did not always follow the rules of the reconstruction ladder but instead adjusted to the patient's situation and reconstruction based on individual conditions and needs. We perform ALT (anterolateral thigh) free flap for squamous cell cancer of the tongue as a choice of preference despite using radial forearm free flap to avoid more morbidity to the patients (Figure 1)

\section{Local recurrence $\&$ status of survival}

After one year of follow-up, five patients (3\%) were declared to have local recurrences. Moreover, based on the survival status variable, we found that twentytwo patients $(12.6 \%)$ died within at least one year after the initial surgery (Table 1 ). 
Table 1. Characteristics of the patients

\begin{tabular}{|c|c|c|}
\hline Characteristics & $\mathbf{n}$ & $\%$ \\
\hline $\begin{array}{l}\text { Age (years), mean } \pm \text { SD } \\
\text { Gender }\end{array}$ & Gender & \\
\hline Male & 49 & 27.9 \\
\hline Female & 125 & 72.1 \\
\hline \multicolumn{3}{|l|}{ Comorbidity } \\
\hline Anemia & 116 & 66.7 \\
\hline Hypertension & 41 & 16.7 \\
\hline Type II diabetes mellitus & 17 & 10 \\
\hline \multicolumn{3}{|l|}{ Primary tumor site } \\
\hline Breast & 76 & 43.7 \\
\hline Head and Neck & 44 & 25.3 \\
\hline Thyroid & 2 & 1.1 \\
\hline Skin & 27 & 15.5 \\
\hline Soft tissue & 25 & 14.4 \\
\hline \multicolumn{3}{|l|}{ Cancer stage } \\
\hline Locally advanced & 131 & 75.2 \\
\hline Metastases & 31 & 17.8 \\
\hline Early stage & 12 & 7 \\
\hline \multicolumn{3}{|l|}{ Types of flap } \\
\hline Local flap & 97 & 55.6 \\
\hline Regional flap & 2 & 1 \\
\hline Distant flap & 16 & 9 \\
\hline Free flap & 60 & 34.4 \\
\hline \multicolumn{3}{|l|}{ Local recurrence } \\
\hline Yes & 5 & 3 \\
\hline No & 169 & 97 \\
\hline \multicolumn{3}{|l|}{ Status of survival } \\
\hline Alive & 152 & 87.4 \\
\hline Dead & 22 & 12.6 \\
\hline
\end{tabular}

Table 2. Types of surgery

\begin{tabular}{lll}
\hline Types of surgery & $\mathbf{n}$ & \% \\
\hline Mastectomy & 73 & 42.1 \\
Breast conserving surgery (BCS) & 3 & 1.7 \\
Wide excision & 54 & 31.1 \\
Compartment resection & 4 & 2.3 \\
Debulking & 5 & 2.9 \\
Total parotidectomy & 2 & 1.1 \\
Extended parotidectomy & 2 & 1.1 \\
Hemiglossectomy & 7 & 4 \\
Total glossectomy & 3 & 1.7 \\
Subtotal glossectomy & 3 & 1.7 \\
Hemimandibulectomy & 9 & 5.2 \\
Subtotal mandibulectomy & 1 & 0.6 \\
Hemimaxillectomy & 3 & 1.7 \\
Partial maxillectomy & 1 & 0.6 \\
Hemimaxillectomy and mandibulectomy & 2 & 1.1 \\
Total thyroidectomy & 2 & 1.1 \\
\hline & & \\
\hline
\end{tabular}

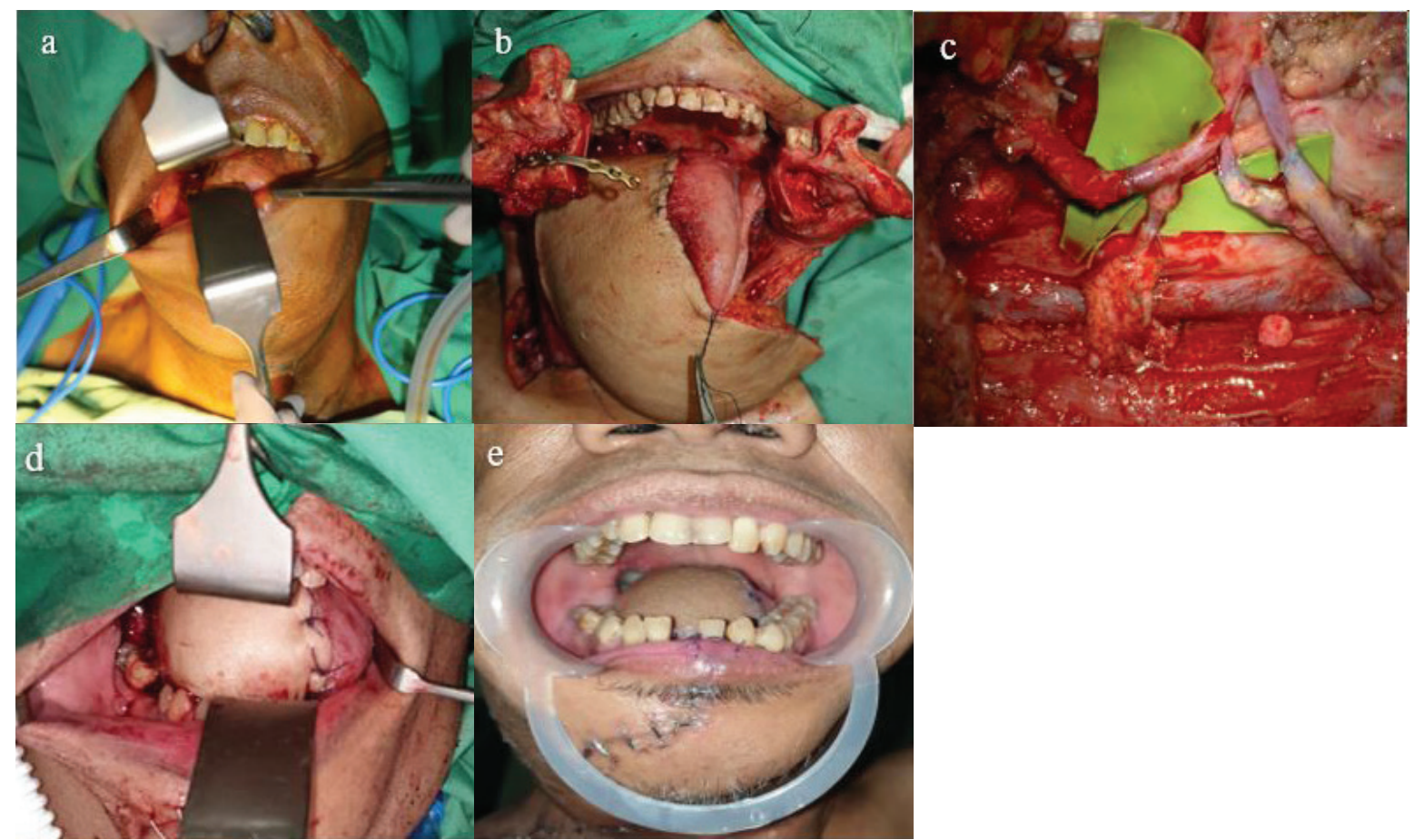

Figure 1. A 46-year-old patient presented with locally advanced squamous cell carcinoma in the tongue. Subtotal glossectomy and neck dissection was done and immediately followed by microsurgery reconstruction using Anterolateral Thigh (ALT) Free Flap. a. preoperative views. b. subtotal glossectomy. c. facial artery and jugular vein anastomosis. d. postoperative views with the outcome at 2 weeks after the surgery. 


\section{DISCUSSION}

Ninety-three percent of patients showed up at our institution in the state of advanced-stage cancer. $75.2 \%$ were in a locally advanced stage, whereas $17.8 \%$ had metastasized. Our institution is a national cancer referral center, where patients have already received many medical treatments in their home regions, coming from different regions and provinces. In addition, long distances can also cause delays in treatment. This situation certainly makes surgery more difficult because patients often have a high tumor burden, which often infiltrates the important structures around it, while we have to be aggressive in cutting.

In our study, breast cancer was ranked first in terms of the primary tumor site. This finding is consistent with data from the Global Cancer Observatory (Globocan) 2020 [6], which stated that breast cancer is the number one cancer in the Indonesian female population. As shown in the results section, females constituted 125 patients in this study (72.1\%). In contrast, male patients made up $27.9 \%$ of the total. The mean age of patients in this study was $48.2 \pm 16.7$ years old.

Moreover, anemia was the most common comorbidity in this study $(66.7 \%)$. The cause of anemia in patients with solid cancer is multifactorial [7]. The patient's malignant wound may be one of the causes. The malignant wound is a manifestation of cancer infiltration into the skin beneath the tumor [5]. The malignant wound usually occurs in patients with a large primary tumor or in patients with a locally advanced stage [7], which made up $75.2 \%$ of the cases at our institution. Malignant wounds bleed easily and are malodorous [5]. As a result, patients must change the gauze regularly. Considerable bleeding can also occur with each gauze change. Cancer-related anemia could also be a consequence of chronic inflammation occurring in patients with advanced cancer and the side effects of myelotoxic agents [7].

In patients with malignant disease, wide excision was performed in fifty-four subjects to remove the tumor entirely and the adjacent tissues surrounding it. We excised for a minimum of one-to-two centimeters from the induration of the primary tumor, aiming to achieve at least a $5 \mathrm{~mm}$ microscopic margin of normal tissue clearance beyond the tumor on the frozen section [8]. Regarding this, wide excision is the main principle of the surgical eradication of most solid malignant tumors [9]. Given that the patients we treated were primarily patients with locally advanced disease, this kind of patient usually had a high tumor burden. In our institution, some cases required more than $2 \mathrm{~cm}$ free margin from the induration of the tumor. A wide excision can leave a defect large enough to require reconstructive action, likewise with breast removal procedures performed on patients with breast cancer.
A mastectomy generally leaves a large defect that requires reconstruction of the chest wall up to the breast itself.

Meanwhile, in head and neck cancer, especially those that have infiltrated the bone and surrounding structures, wide excision often must be accompanied by removing the organ of the origin of the primary tumor, either partially or entirely. Procedures such as subtotal glossectomy and hemimandibulectomy are also performed to ensure that the tumor can be removed en bloc [9].

Among all patients, ninety-seven patients (55.6\%) received local flaps as a reconstruction method. The type of flap given to the patient depends on the operator's choice and the defect's shape, but at our institution, many types of local flaps have been applied, including advancement flap, transpositional flap, and rotational flap. One patient received a nasolabial flap after removal of the squamous cell carcinoma of the nasal cavity. Meanwhile, for chest wall defects caused by mastectomy, apart from rotational flaps, we also often used obliquus externus musculocutaneus (OEM) flaps and keystone and double keystone flaps as local advancement flaps. The local flap has the advantage of providing tissue of the same quality as the defect area tissue under normal conditions. It lowers the likelihood of flap failure and necrosis [2].

Aside from the local flap, a regional flap can also be selected, which means that the tissue is taken from outside the local area but is still in a nearby anatomical region, as in the connotation of using the term locoregional [2]. Two patients (1\%) received regional flaps. One patient received a sural flap after removal of lower limb sarcoma tumors. Meanwhile, another patient received a forehead flap after wide excision of basal cell carcinoma in the nasal region.

Given the limited local tissue availability and multiple tissue requirements, many breast reconstruction techniques required a distant flap performed in sixteen patients (9\%). Donor sources can come from the lower abdominal region or the back region [11,12]. One of the most frequently applied distant flaps in our institution was the trans-rectus abdominis musculocutaneous (TRAM) flap and latissimus dorsi (LD) flap.

Meanwhile, free flap, also known as free tissue transfer, which requires microsurgery, was done in sixty patients (34.4\%). The free flap technique is beneficial for reconstructive surgery as it enables the harvesting of a large amount of revascularized tissue, and it can be tailored to the defect and allows for more complex reconstructive procedures, which in turn permit more extensive resections [12]. It can result in fewer patients requiring further re-excision and decreased local disease recurrence in the long term [8]. Free flap donors can be taken from all body regions. Any axial flap from the 
whole body, in theory, and practice, can be elevated to a free flap. An example is the latissimus dorsi axial flap, which can become a musculocutaneous free flap with the thoracodorsal vascular pedicles [10]. There were three of our patients who received an LD-free flap (5\%). Anterolateral thigh (ALT) free flap was the type of flap that we most often applied to patients because the pedicle dissection of blood vessels is relatively easy and familiar. Also, the ALT flap usually consists of skin, subcutis, and fascial tissue, so that it is quite thick. It is beneficial for reconstructing structures that require volume or bulk. The ALT-free flap was performed in 25 patients (41.7\%). Meanwhile, groin flap, also known as the superficial circumflex iliac artery perforator (SCIP) flap, is widely applied in reconstruction post-removal of tumors in the head and neck area [12]. This flap could also provide the bulk required for oral cavity reconstruction and hence was performed in 21 patients (32.8\%).

In the process of reconstructing structures such as the maxilla and mandible, other materials such as plates and screws and the use of vascularized bone grafts are required. The bone used as a donor, in this case, is the fibular bone. The diaphysis of the fibular bone is cut and removed as a vascularized bone, with the pedicle, i.e., the peroneal artery. The peroneal artery has five to six branches along the fibular bone. This condition allows the fibular bone to be cut into several short segments to be shaped to follow the contour of the bone to be reconstructed. The fibular flap material is the primary choice for mandibular arch reconstruction [9]. Free fibular flaps were performed in six of our patients (3.4\%) who had undergone hemimandibulectomy or subtotal mandibulectomy.

Apart from being a surgeon that performs reconstruction, a surgical oncologist must also act as an oncologist who ensures that the patient is tumorfree and determines further treatment $[13,14]$. As the primary physician who treats the tumor from the start, a surgical oncologist is also more reliable in detecting local recurrence when it does occur [13]. Knowledge as a particular oncologist will significantly assist in choosing the flap or reconstruction methods to be given; for example, we rarely performed a skin graft on patients deemed to have radiation therapy because the radiation can adversely affect skin healing. Furthermore, because the surgeon performing tumor eradication and reconstruction is the same person, the surgeon can preserve structures carried out in later reconstruction, especially vital structures such as the vascular and nerve supply.

Moreover, anemia was the most common comorbidity in this study $(66.7 \%)$. The cause of anemia in patients with solid cancer is multifactorial [7]. The patient's malignant wound may be one of the causes. The malignant wound is a manifestation of cancer infiltration into the skin beneath the tumor [5]. The malignant wound usually occurs in patients with a large primary tumor or in patients with a locally advanced stage [7], which made up $75.2 \%$ of the cases at our institution. Malignant wounds bleed easily and are malodorous [5]. As a result, patients must change the gauze regularly. Considerable bleeding can also occur with each gauze change. Cancer-related anemia could also be a consequence of chronic inflammation occurring in patients with advanced cancer and the side effects of myelotoxic agents [14][7].

Within one year of follow-up, five patients (3\%) were found to have a local cancer recurrence. All of them had head and neck cancer and were diagnosed in a locally advanced stage. In addition, the type of cancer these patients had was histopathologically aggressive or known as high-grade. We also found out that there was a delay in adjuvant treatment prescribed to patients. Four of the patients did not undergo adjuvant therapy, specifically radiotherapy, in less than six weeks due to personal reasons. It might contribute to cancer recurrence.

The disease-specific survival rate among all patients was $87.6 \%(n=152)$ after a year of follow-up. Of those lost to follow-up due to death, the cause of mortality was not directly attributed to the operative procedure. Although there were no similar data from our institution regarding locoregional recurrence and survival status of patients undergoing standard surgical techniques rather than oncological reconstructive surgery, a meta-analysis from an institution in London showed no significant difference between the outcomes of oncoplastic breast surgery compared to traditional techniques. This study included two variables: cancer recurrence and reoperation due to positive margins or borderlines [4]. Besides, one retrospective study conducted at MD Anderson Cancer Center in patients with advanced oral cavity cancers found that survival and recurrence rates between those who had undergone oncological reconstructive surgery and those who had not did not differ significantly. However, the introduction of oncological reconstructive surgery, specifically with free flaps, enables patients with a higher tumor burden (T4) and more advanced $\mathrm{N}$ classification to be treated. Positive margin rates also decreased significantly [5].

Further study with more prominent participants is suggested with a more evident algorithm for choosing the reconstruction method for each patient. In this study, the type of reconstruction performed on the patient was based on the operator's judgment. Hence, it might introduce a bias affecting the outcome in the group of participants. 


\section{CONCLUSIONS}

Oncological reconstructive surgery is a new approach in the field of surgery and may confer additional benefit aside from removing the tumor, as it serves a reconstructive function. Further studies are required to evaluate the extent of the benefits and risks conferred by this new method.

\section{DECLARATIONS}

\section{Ethics Approval and Consent to Participate}

The ethical clearance was obtained from the ethics commission of health research in Dharmais National Cancer Center with the Number 160/KEPK/XII/2020.

\section{Competing of Interest}

The authors declare no competing interest in this study.

\section{Research Funding}

This research had no sponsorship and was fully funded by the researchers.

\section{Acknowledgment}

The authors acknowledge the support from the research staff at our department, and also from the Hospital.

\section{REFERENCES}

1. Van Paridon MW, Kamali P, Paul MA. Oncoplastic breast surgery: achieving oncological and aesthetic outcomes. J Surg Oncology. 2017;116(2):195-202.

2. Piper M, Peled AW, Sbitany H. Oncoplastic breast surgery: current strategies. Gland Surg. 2015;4(2):154-63.

3. de Vicente JC, Rodríguez-Santamarta T, Rosado P, et al. Survival after free flap reconstruction in patients with advanced oral squamous cell carcinoma. J Oral Maxillofac Surg. 2012;70(2):453-9.

4. Mansell J, Weiler-Mithoff E, Stallard S, et al. Oncoplastic breast conservation surgery is oncologically safe when compared to wide local excision and mastectomy. Breast. 2017;32:179-85.
5. Hanasono MM, Friel MT, Klem C, et al. Impact of reconstructive microsurgery in patients with advanced oral cavity cancers. Head Neck. 2009;31(10):1289-96.

6. International Agency for Research on Cancer. Globocan, Indonesia number of new cases 2020 [Internet]. International Agency Research of Cancer. 2021 [cited 2021 Jun 2]. Available from: https:// gco.iarc.fr/today/data/factsheets/populations/360indonesia-fact-sheets.pdf

7. Gao F, Cheng K, Zhao F, et al. Prevalence and characteristics of anemia in patients with solid cancers at diagnosis in southwest China. Asian Pac J Cancer Prev. 2011;12(11):2825-8.

8. Edwards SP. Margin analysis-has free tissue transfer improved oncologic outcomes for oral squamous cell carcinoma? Oral Maxillofac Surg Clin North Am. 2017;29(3):377-81.

9. McCrory AL, Magnuson JS. Free tissue transfer versus pedicled flap in head and neck reconstruction. Laryngoscope. 2002(12);112:2161-5.

10. Liang J, Yu T, Wang $X$, et al. Free tissue flaps in head and neck reconstruction: clinical application and analysis of 93 patients of a single institution. Braz J Otorhnolaryngol. 2018;84(4):416-25.

11. De Bree R, Rinaldo A, Genden EM, et al. Modern reconstruction techniques for oral and pharyngeal defects after tumor resection. Eur Arch Otorhinolaryngol. 2008;265(1):1-9.

12. Chatterjee A, Dayicioglu D, Khakpour N, Czerniecki B. Oncoplastic surgery. Cancer Control. 2017;24(4):3-5.

13. Losken A, Dugal CS, Styblo TM, Carlson GW. A metaanalysis comparing breast conservation therapy alone to the oncoplastic technique. Ann Plas Surg. 2014;72(2):145-9.

14. Haloua $M H$, Krekel NM, Winters $H A$, et al. A systematic review of oncoplastic breast-conserving surgery: current weaknesses and future prospects. Ann Surg. 2013;257(4):609-20. 